\title{
ASPECTOS ETICOS EN LA INVESTIGACION CIENTIFICA
}

\author{
ETHICAL ISSUES IN CIENTIFIC RESEARCH \\ IRENE ACEVEDO PÉREZ ${ }^{1}$
}

\begin{abstract}
RESUMEN
El escrito pretende mostrar los diferentes códigos, declaraciones y normas que se han dictado en el mundo para la protección de los seres humanos sometidos a experimentación científica. Se analiza el tema a la luz de los principios éticos y la situación actual en Chile en relación a la existencia de los comités de ética científicos.
\end{abstract}

Palabras claves: Experimentación en seres humanos, Principios bioéticos, Protección.

\begin{abstract}
The article's aim is to show all different codes, declarations and norms that have been dictated in the world, in order to protect the human beings submitted to scientific experimentation. The topic is analyzed considering ethical principles and the present situation in Chile, related to the existence of the scientific ethical committees.
\end{abstract}

Keywords: Experimentation in human beings, Bioethical principles, Protection.

Los progresos científicos y tecnológicos que día a día aportan el conocimiento de modernos procedimientos y avances en la medicina, por una parte, conllevan un sinnúmero de beneficios para las personas sanas o enfermas; pero, por otra, surgen de las investigaciones que se realizan en seres humanos diversos dilemas éticos, como producto, en determinadas ocasiones, del no cumplimento de normas, códigos o reglamentación en la investigación experimental.

Si bien es cierto la experimentación en seres humanos tiene como propósito contribuir al mejoramiento de procedimientos diagnósticos y profilácticos y además la comprensión de la etiología y patogenia de una enfermedad, no es menos cierto que estos protocolos deben pasar por un cauteloso filtro que proteja los derechos de los probandos $\mathrm{y}$, en especial, de aquellas poblaciones vulnerables donde pudiera ser violentada la dignidad de la persona humana.

Las normas éticas han expresado que los experimentos deben hacerse primariamente en animales y, según los efectos por analogía, se podrían emplear en seres humanos. Paul Ehrlich plantea la tesis que no es suficiente el principio de analogía para conocer cuál es el comportamiento de los fármacos en los seres humanos, sino que habría que ensayar en seres humanos para conocer las propiedades y el comportamiento farmacológico de los productos terapéuticos. Bradford Hill definió el método del ensayo clínico como "un experimento cuidadoso y éticamente diseñado, con el fin de poder contestar a preguntas concretas formuladas previamente". Esta nueva mirada obliga a los investigadores a construir diseños que respalden la verdadera investigación clínica (5).

En el año 1947 la enunciación del Código de Nuremberg marcó una de las primeras medidas de protección de las personas humanas en pro del ejercicio de la autonomía; en él se señala que el consentimiento voluntario es esencial y que, en ausencia de la posibilidad de ejercerla, si fuere necesario, deberá existir un representante legal; no po-

\footnotetext{
${ }^{1}$ Académico Escuela de Enfermería. Facultad de Medicina. Universidad de Chile. Magíster en Bioética. Especialista en Enfermería Pediátrica.
} 
drá haber coacción, fraude, engaño o presión sobre los probandos. Obliga al investigador a proporcionar una información comprensible relacionada con la naturaleza, la duración, el propósito, el método utilizado, las molestias, los inconvenientes, los daños y los efectos en la salud de las personas que participen en un protocolo de investigación. En este código se espera que los experimentos obtengan buenos resultados para la comunidad, que se estudie la historia natural de la enfermedad y que debe evitarse el sufrimiento físico y mental de las personas (1).

Señala, además, este código que no deberán realizarse experimentos en situaciones de riesgo de muerte o de daño incapacitante y que el grado de riesgo no debe exceder el grado de importancia del experimento. Destaca la protección del daño, la incapacidad o la muerte; cautela la dirección de los protocolos por científicos experimentados, que el sujeto tenga la libertad para retirarse en el momento que lo desee y, por último, que el investigador debe asumir que en cualquier momento puede terminar su estudio.

En 1964 aparece el pronunciamiento señalado en la Declaración de Helsinki, el cual entra en vigencia el año 1989. En ella se declara que la finalidad de la investigación biomédica con sujetos humanos debe ser el "mejoramiento de los métodos diagnósticos, terapéuticos y profilácticos y el conocimiento de la etiología y la patogenia de la enfermedad". Sus principios se centran en aspectos como los que se señalan a continuación: que los trabajos deben ajustarse a los principios científicos y basarse en experimentos y estudios en animales; que el diseño y la ejecución debe formularse en un protocolo experimental y que deberá ser revisado por un comité independiente; que los trabajos deben ser conducidos por expertos; que la importancia del objetivo a alcanzar debe ser mayor a los riesgos de la investigación; que deberán evaluarse los riesgos previsibles y los beneficios tanto para el sujeto como para otras personas; que es fundamental respetar el derecho a salvaguardar la intimidad y la integridad personal; que sólo deberán reali- zarse investigaciones cuando los riesgos inherentes sean previsibles; que es preciso respetar la exactitud de los resultados en la publicación y que no deberá existir coacción en la obtención del consentimiento informado. Esta Declaración se amplió el año 2000, al considerar fundamentalmente los aspectos relacionados con la experimentación que utiliza placebos (3).

El artículo $7^{\circ}$ del Pacto Internacional de Derechos Civiles y Políticos, aprobado por la Asamblea General de las Naciones Unidas, señala: "Nadie será sometido a torturas o tratos crueles, inhumanos o degradantes. En particular, nadie será sometido sin su libre consentimiento a experimentos médicos o científicos".

En 1982 la publicación de las Normas Eticas Internacionales para la Investigación Biomédica con Sujetos Humanos pretende destacar la protección de las comunidades más vulnerables, aportando para cada grupo de edades o de determinadas patologías, la seguridad que sus derechos serán respetados (2).

En Chile, el año 1997, el Ministerio de Salud convocó a un grupo de expertos al estudio de los diferentes códigos existentes en el mundo, con el fin de regularizar la ejecución de ensayos clínicos que utilizan medicamentos en seres humanos. Si bien es cierto en alguna medida existían normativas tales como los artículos $1^{\circ}, 2^{\circ}$ y $19^{\circ}$ de la Constitución Política del Estado, relacionados a diversos derechos de la personas, el artículo $102^{\circ}$ del Código Sanitario y el artículo $16^{\circ}$ letra c) del Decreto Supremo 1.876/95, que señala que le "corresponde al Director del Instituto de Salud Pública de Chile otorgar la autorización para el uso provisional de productos farmacéuticos para fines de investigaciones científicas o ensayos clínicos, sin registro previo y mediante resolución", se describe también la reglamentación existente en los campos clínicos a través de la reglamentación de los Servicios de Salud respecto a la autorización que los directores de hospitales deben dar a los proyectos de investigación y a la creación de Comités de Etica para la supervisión de éstos. 
$\mathrm{Al}$ respecto, se puede señalar que en 1994, la resolución exenta $\mathrm{N}^{\mathrm{o}} 134$ del Ministerio de Salud aprueba la existencia de Comités de Etica Médica, los que deberán "tratar las situaciones en que estén involucradas decisiones sobre procedimientos diagnósticos y terapéuticos de alto costo o controversiales".

El año 2001 el Ministerio de Salud capacitó y acreditó en Chile los primeros Comités de Etica Científicos, cuyo propósito es contribuir a salvaguardar la dignidad, los derechos, la seguridad y el bienestar de todos los participantes actuales y potenciales de la investigación; los principios que comandan estos comités dicen relación con "el respeto a la dignidad de la personas y la consideración al principio de la justicia". Deben ser equipos multidisciplinarios y multisectoriales, con independencia de influencias políticas, institucionales y comerciales, con competencia y eficiencia en su trabajo para la evaluación de la investigación propuesta.

Estos comités basan su actuar en la Declaración de Helsinki, en las Guías Eticas internacionales para la Investigación Biomédica, en las Guías para la Buena Práctica Clínica, en guías nacionales y locales, en las Normas Eticas para la Regulación de Experimentación en Seres Humanos y en los Principios Bioéticos. Cada revisión de un protocolo contempla el análisis de la naturaleza y los objetivos de la investigación, su fundamento científico, la metodología que se usará en la recolección y el procesamiento de los datos y la competencia del investigador.

Desde el punto de vista del principio de justicia, se constata el riesgo mínimo para el probando, se contrasta el riesgo con el beneficio que se obtendrá y la moralidad del placebo. Se analizan los sistemas de selección equitativa de los sujetos, en especial la protección de los grupos más vulnerables y la compensación que tendrán los daños que se puedan presentar. El principio de autonomía debe contemplar la revisión acuciosa y responsable del documento de consentimiento informado, considerando aspectos que dicen relación con los niveles de información, los niveles de consentimiento, las decisiones de sustitución, la protección a la intimidad y confidencialidad de los datos y se comprueba además cómo se analizarán los resultados. Una vez revisada toda esta información, los componentes de este comité proceden a votar la aprobación, la aprobación posterior a las enmiendas sugeridas o el rechazo definitivo del protocolo. Para la revisión y el análisis de los proyectos, los integrantes basan su informe en un documento que detalladamente permite evaluar los antecedentes de cada protocolo. Se deberán reportar los eventos adversos, señalar periódicamente los avance de los proyectos y los resultados finales obtenidos.

En la investigación donde se experimente con seres humanos, se hace fundamental la aplicación de los principios de beneficencia, autonomía, justicia y no maleficencia. El principio de autonomía, bien llamado también respeto por las personas, se basa en el fundamento de que el ser humano posee la capacidad de darse a sí mismo su actuar como persona, es decir, determinar su propia norma; autónomamente tiene la libertad de elegir, aplicando su propio razonamiento y una vez analizados los aspectos negativos y positivos, determinará qué conducta seguir. Kant señala que el hombre es persona por la capacidad que tiene de darse a "sí mismo el imperativo categórico de la ley moral"; una persona ejerce su autonomía cuando no es influenciado por personas o circunstancias (6).

Faden y Beachamp señalan que un acto elegido autónomamente debe cumplir con tres condiciones: ausencia de control externo, intencionalidad y conocimiento. Se entendería por ausencia de control externo la manipulación, la coerción y la persuasión; definen manipulación como "la influencia intencional y efectiva de una persona por medios no coercitivos, alterando las elecciones reales al alcance de otra persona, o alterando por medios no persuasivos la percepción de esas elecciones por la persona". Persuasión es la influencia con intención de inducir a aceptar libremente valores o creencias o actitudes del persuasor. Existiría coerción cuando se ejerce influencia intencio- 
nal y efectiva y bajo amenazas de provocación de daños no deseados y que se pueden evitar.

La intencionalidad existe cuando es querida o se desea y el conocimiento es señalado como el nivel o grado de entendimiento o comprensión de la acción, la cual debe ser completa y adecuada. La experimentación en los seres humanos debe, básicamente, respetar el principio de autonomía a través de la aplicación del consentimiento informado.

El principio de beneficencia persigue maximizar los beneficios y minimizar los daños, por tanto los participantes en una investigación o sus representantes deben conocer los riesgos y los beneficios que lograrán con su participación en los ensayos clínicos; se deberá alcanzar que los riesgos sean mínimos asociados a los máximos beneficios que se conseguirán. El poder que puede ejercer el probando a través del ejercicio de su autonomía en la decisión de participar, rompe el modelo del tradicional paternalismo. Nos enfrentamos entonces, nuevamente, a un nuevo dilema ético, es decir, la capacidad de decisión de las personas que participan en un proyecto de investigación y quienes ejercen la autonomía en caso de tratarse de un niño o un mentalmente incapaz.

Cuando asociamos principio de justicia con la investigación científica, podemos aseverar que lo justo se identifica con lo bueno y lo correcto: Es esperado, por tanto, en este contexto que todas las personas sean beneficiadas con los resultados de los experimentos, que se realicen realmente en los grupos que se requiere investigar y que sólo se utilicen las poblaciones vulnerables cuando en éstas sean beneficiosas las consecuencias. Se buscará sin duda la equidad y la óptima distribución de recursos en pos de una excelencia en la investigación científica.

El principio de no maleficencia "obliga a todos de modo primario y por lo tanto es anterior a cualquier tipo de información o de consentimiento" y pretende no dañar al paciente, lo que obligaría moralmente al investigador a buscar los menores riesgos posibles para los sujetos de experimentación. Concre- taremos diciendo que el investigador será el responsable de los principios de no maleficencia y de beneficencia, el probando de ejercer su autonomía y el estado de cautelar el principio de justicia (6).

No es fácil la misión que los Comités de Etica Científica tienen con relación al avance de la ciencia y la tecnología; pueden ser sentidos como trabas para el investigador, pero el mejoramiento del proceso investigativo es la principal tarea que tienen sus integrantes, de tal manera de optimizar el proceso, pero, por sobre todo, de cautelar los derechos de las personas a través del respeto a la dignidad humana.

Enfermería, tímidamente, se va adentrando a la investigación experimental en seres humanos y deberá ser un deber y una obligación moral capacitarse en esta área del conocimiento científico. Para tal efecto, su misión es integrar estos comités, programar investigaciones experimentales con más fuer$\mathrm{za}$, crear documentos de consentimiento informado para diferentes procedimientos o técnicas, crear instrumentos de evaluación y de seguimiento de protocolos y compartir su saber a través de la publicación de los resultados obtenidos. La investigación científica debe ir caminando a la par con el avance de nuestra disciplina, ya que será fundamental su realización y uno de los tantos roles que ejerce día a día el profesional de Enfermería.

\section{REFERENCIAS BIBLIOGRAFICAS}

1. Código de Nuremberg. 1947.

2. Propuestas de normas éticas internacionales para la investigación biomédica con sujetos humanos. 1982.

3. Declaración de Helsinki 1989-2000.

4. Gracia D. Investigación clínica. En Gracia D. Etica y vida. Vol. 4, Profesión Médica, Investigación y Justicia Sanitaria. Bogotá, El Buho. 1998: 77-117.

5. Pellegrini A; .Macklin R. Investigación en sujetos humanos. Experiencia. En Gracia D. Investigación en sujetos humanos: Implicancias lógicas, históricas yéticas, Internacional. Editores O.P.S.- O.M.S. 1999. 63-81.

6. Ministerio de Salud. Normas técnicas de regulación de ensayos clínicos en seres humanos. Chile. 2000. 